\title{
Numerical Geodesic Approximation for Theoretical and Experimental Light Bending Analysis
}

\author{
Robert L. Shuler \\ NASA Johnson Space Center, Houston, Texas, United States \\ Email: robert.1.shuler@nasa.jsc or robert@mc1soft.com
}

\begin{abstract}
This paper investigates a least-time (or fastest-path) two-point algorithm for numerically propagating a light ray in a gravitational field using anisotropic coordinate velocity and distantobserver coordinates. Rather than imaging or ray tracing, the objective is to support analysis of fundamentals and to be able to find null geodesics in arbitrary metrics. First we establish that results agree with geodesic paths in regard to bending angles to very high accuracy. Then we investigate the bending rate of two coordinate bending angles, wave and displacement, and find that wave angle illustrates well-known points about spatial and time components of light bending, while the displacement angle leads to simple analytic description of Einstein's comment about the relation between these components, and a new figure showing the compatibility of light bending and equivalence. A second analysis compares light bending near a neutron star for Schwarzschild and one alternate metric to evaluate feasibility of future experiments. Two methods of extension to non-light speed objects are discussed.
\end{abstract}

Keywords: gravity; light bending; space-time; curvature; equivalence; general relativity

\section{Introduction}

In this paper we begin by introducing an effective and accurate computational technique for calculating light paths in a static spherically symmetric gravitational field using only simple mathematics, the local constancy of the speed of light from Special Relativity (SR), the principle of coordinate velocity, and the Schwarzschild line element from General Relativity (GR). It is easy to substitute other metrics as long as they are static. Unlike numerical methods which propagate and store a family or group of geodesics efficiently for purposes such as imaging, [1] [2] or handle the fields of rotating or collapsing objects, [3] this method attempts to propagate a single ray in an easy-to-visualize metric for purposes of identifying patterns and behavior relevant to the investigation of fundamental or experimental issues. First the method is described and its accuracy is established, then two questions are studied.

The first question is an old one, the relation between so-called "double bending" and equivalence. In his 1916 book, Einstein said, "According to the theory, half of this deflection is produced by the Newtonian field of attraction of the sun, and the other half by the geometrical, modification ('curvature') of space caused by the sun. " [4] We take the discussion of Ehlers and Rindler regarding the locality of bending relating to time dilation and the inability to localize bending related to the spatial component of the metric as the current "best explanation." [5] The baseline for single or time dilation bending via equivalence (resulting in a lower coordinate velocity and refraction bending) is taken as that in Einstein 1911 [6] and assumed to be what he meant by "field of attraction," since time dilation was inferred from universal acceleration in that source. For full and correct or "double" bending, i.e. both halves, we take Einstein 1916, [7] and for $2^{\text {nd }}$ order full bending, Bodenner and Will[8]. The algorithm is able to plot either type of bending by adjusting coordinate velocities. Results will include comparative plots of two coordinate angles, one a wave angle which illustrates the varying "location" of full vs. equivalence bending, and the other an interesting coordinate displacement angle comparison which only approaches the total bending angle asymptotically and therefore doesn't give "location," but which shows a displacement doubling point by point. Two figures new to the literature support analytical verification of the coordinate displacement doubling, and show how spatial-component bending is excluded from an equivalence frame, preserving compatibility with equivalence regardless of location of bending. 
The second question is an investigation of light bending near a dense object such as a neutron star, comparing General Relativity (GR) with another arbitrarily selected metric differing only at $2^{\text {nd }}$ order to assess the feasibility of certain kinds of $2^{\text {nd }}$ order GR field strength tests (as opposed to for example order in $v / c$ which has already been tested to $3^{\text {rd }}$ order [9]) in astrophysical settings.

\section{$2 \quad$ Methods}

\subsection{Coordinates}

We perform computations in a global distant-observer Cartesian Euclidean space $\mathbb{R}^{2}$ which at large radii $r$ coincides with the observer space, so that angles at large radii require no transformation. The mass of interest $\mathrm{M}$ resides at coordinates $(0,0)$ which is taken as the origin of a coincident static Schwarzschild coordinate space for the purpose of computing the vector coordinate velocity of light at points in $\mathbb{R}^{2}$. As long as M's rate of spinning is limited and other mass distributions are not presently at sufficient density to deflect the light by greater than our margin of computational error, the light ray can be considered to stay in this plane.

Such coordinates do not take advantage of symmetry for analytical problems, and require complex measures to handle singularities and retain accuracy in numerical methods. However, for visualizing and diagramming they naturally fit our minds and pages, and for computational models they provide a global coordinate framework to track state vectors which is independent of the positions and masses of objects. But since the coordinate distance is dependent on coordinate velocity, which varies with position, vector operations are limited to local regions in which the coordinate velocity can be approximated as a constant. Propagation via locally valid displacement vectors is simple vector addition. The question of light bending arises within such coordinates as planetary observers see no change in relative angular position of stars beyond the parallax range except when they pass near the central solar mass.

\subsection{Static Spherically Symmetric Field Metric}

Writing the Schwarzschild metric as a line element using $\Upsilon=\left(1-2 G M / r c^{2}\right)^{-1 / 2}$ gives:

$$
\mathrm{d} s^{2}=-\mathrm{d} \tau^{2} c^{2}=-\Upsilon^{-2} c^{2} \mathrm{~d} t^{2}+\Upsilon^{2} \mathrm{~d} r^{2}+r^{2}\left(\mathrm{~d} \theta^{2}+\sin ^{2} \theta \mathrm{d} \phi^{2}\right)
$$

We use this metric to determine the time dilation and spatial expansion factor $\Upsilon(r)$. For the rules of light propagation we use the method described in this section, and the $\mathbb{R}^{2}$ Cartesian coordinates $(x=r \cos \theta, y=r \sin \theta)$. To a coordinate stationary observer using coordinates $\mathrm{d} s$ and $\mathrm{d} \tau$, light travels locally at velocity $c$. Our method is to convert this local velocity, taken at two nearby points on the wave front, into coordinate velocities at corresponding points in distant observer Cartesian coordinates $\mathbb{R}^{2}$ using radial and tangential transformations of the light's velocity to coordinate velocity in $\mathbb{R}^{2}$, and integrate it in a Huygens-like fashion.

To calculate $\Upsilon(r)$ from (1), we can set $\mathrm{d} \theta=\mathrm{d} \phi=0$ and use $r=\left(x^{2}+y^{2}\right)^{1 / 2}$. In static fields for clocks that don't move we have $\mathrm{d} r=0$ and therefore $\mathrm{d} \tau=\Upsilon^{-1} \mathrm{~d} t$. Similarly, at an instant in time for any particular observer we have $\mathrm{d} t=0$, and for a displacement $\mathrm{d} s$ constrained to a vertical distance $\mathrm{d} h$ we can write as $\mathrm{d} s_{\text {radial }}=\mathrm{d} h=\Upsilon \mathrm{d} r$. Thus we have relationships between differentials in the metric or distant observer coordinate differentials $\mathrm{d} r$ and $\mathrm{d} t$, and local coordinate differentials $\mathrm{d} \tau$ and $\mathrm{d} h$. Coordinate velocities in this setup have the well-known forms $c_{\text {tangential }}=c / \Upsilon$ due to time dilation, and $c_{\text {radial }}=c / \Upsilon^{2}$ due to the combination of time dilation and spatial expansion. From a unit vector $\mathbf{u}$ in a particular direction at a particular point $\mathbf{p}$ in $\mathbb{R}^{2}$, we resolve to tangential and radial components via a unit vector in the direction of the origin and calculate coordinate velocities using the magnitude of the radius, which will be the same as the Schwarzchild coordinate radius $r=C / 2 \pi$. Multiplying the tangential and radial coordinate velocities of light by the respective components of the unit vector, we obtain the coordinate velocity of light in the direction of $\mathbf{u}$ at $\mathbf{p}$ in $\mathbb{R}^{2}$. 


\subsection{Propagation Method (Numerical Fastest-path Approximation of Fermat's Principle)}

Light propagates as a wave, not as a particle. In principle, we can use the geodesic equations and there is no difference in methodology. In practice, simplifications to the calculations are made by first determining whether the geodesic is time-like (the trajectory of a free falling particle) or null (the trajectory of a light ray). Particles which are for example initially at rest (with respect to the gravitating mass) will experience acceleration. The intuitive introduction to geodesic paths used in one famous text [10] of an ant navigating the surface of an apple obscures this point. The ant is propelling itself. An object seemingly at rest must accelerate. Perhaps one could extend the analogy by considering that the rest object is walking through time, but this difficult abstraction defeats the intuitive value. In practice, time-like geodesics involve acceleration. On the other hand, since the speed of light is constant, changing only in direction, light rays are more perfectly analogous to the ant.

In this paper, we consider only the path of light rays, or null geodesics. In a separate treatment the author hopes to generalize the alternative methods to encompass time-like geodesics, but it is appropriate to address and understand the simpler case first. Without doubt, null geodesics are simpler. Consider that as a particle-like object moves faster and faster, approaching the speed of light, more of its energy is light-like and a smaller and smaller fraction is its particle-like rest energy. In this limit, null geodesics are approached by time-like geodesics. It is our hypothesis that we can successfully understand null geodesics as the motion of light through curved space-time, much like the motion of the ant on the apple.

It is our further hypothesis that to construct a simple and efficient propagation algorithm, we can use a least-time or fastest-path approach, in other words Fermat's principle, which has the advantage of being coordinate free[11]. In a perfect implementation each point of a wave front is propagated in all possible directions for a small $\Delta t$ interval. The tangent to the infinitely many circles defined by all possible propagations represents the farthest points reachable in $\Delta t$. The wave does not include backward points (except from the source point) because those have already been reached at an earlier time. We can modify Huygens' Principle to accommodate asymmetric propagation velocity and still find the least-time new wave front as shown in Figure 1. To compute the path of a light ray, we only need two points on a wave front propagated in the Huygens manner, a differential distance apart with magnitude of the distance chosen by numerical considerations. The optimum new wave front, shown in the figure by a heavy dashed line, would be tangent to ovals generated by coordinate velocity vectors propagated in all directions from the end points over some coordinate time interval $\mathrm{d} t$. A reduction in computation is obtained by computing only one propagation vector for each point, normal to the old wave front, and taking the new wave front as a line between the new end points, shown as the small dotted line in the figure. Bending in this approach comes from the new angle of the new wave front.

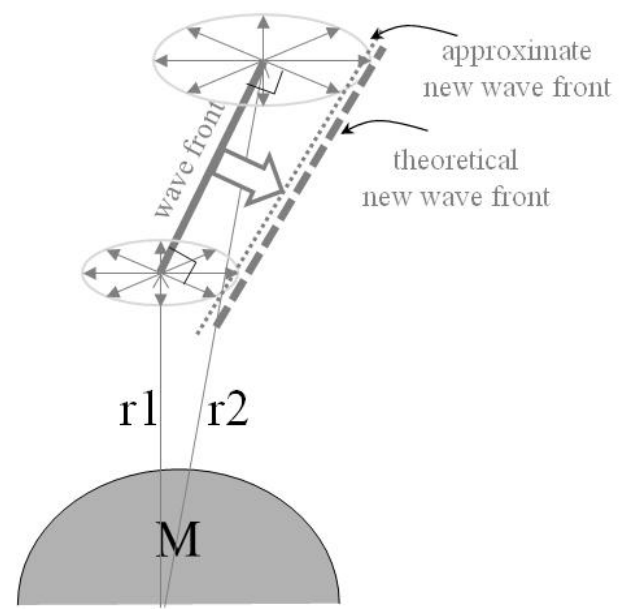

Figure 1. Least-time path construction via 2-point approximation 


\subsection{Implementation Parameters}

To evaluate the computational model, C language versions in long double (80 bit) and GCC Quad Precision (128 bit) were implemented, and for reader interaction a Javascript web page (limited to 64 bit double precision) can be found at http://mc1soft.com/papers/lightbending/. The parameters of the script are shown in Figure 2. The web version defaults to parameters which represent a light ray passing at the solar limb, with a 200 kilometer separation of propagation points on the wave front (chosen for Huygens' tangent approximation validity, not to imply light actually passing through the sun), and a time step of one millisecond in observer coordinate time, during which the light will travel approximately 300 kilometers. Computational data is displayed in a new window or tab at a specified display interval, and can be copied into a spreadsheet for graphing or further analysis. ${ }^{1}$

Since the two halves of the light path will be symmetric, the script only propagates the light from the solar limb, on an initially tangent trajectory, to the selected stopping radius or time, and doubles this amount to determine end to end bending. Values for a particular stopping point sufficiently large, e.g. 10 times Earth's orbital radius, i.e. 10 AU (astronomical units), will differ only slightly from analytically obtained bending. Options are provided to run some experiments regarding "what if" the coordinate velocity were different, for example determined only by time dilation, so that a comparison similar to Brown's can be made.

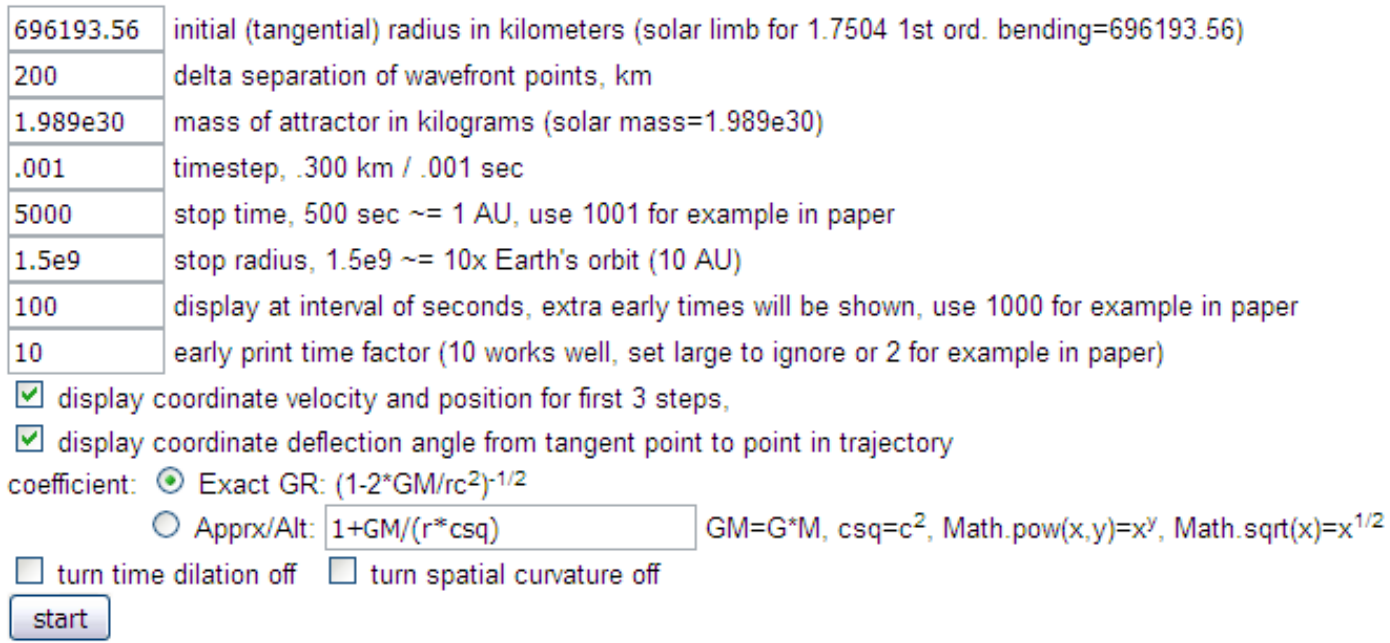

Figure 2. Computational light bending script parameters.

\subsection{Displacement and Wave Angle Definitions}

If the initial tangent point for purposes of computing the half angle is $\mathbf{p} \mathbf{0}=(\mathrm{x} 0, \mathrm{y} 0)$ and the propagating wave points are $\mathbf{p} \mathbf{1}=(\mathrm{x} 1, \mathrm{y} 1)$ and $\mathbf{p} \mathbf{2}=(\mathrm{x} 2, \mathrm{y} 2)$, then define the center point of the wave segment $\mathbf{p c}=$ $(\mathbf{p} 1+\mathbf{p} 2) / 2$. The simulation will print the bending angle in two ways, as a wave front angle which is a 90 degree rotation of the vector from $\mathbf{p} \mathbf{1}$ to $\mathbf{p} \mathbf{2}$, illustrated as angle $\boldsymbol{\omega}$ in Figure 3 . The displacement angle is in the direction of a vector from the tangent point p0 to the location pc, with two illustrations $\boldsymbol{\delta}_{1}$ and $\boldsymbol{\delta}_{2}$ in the figure. Both are coordinate quantities. Since at large radii (right side of figure) the coordinate system matches approximately flat local observer space, the wave angle is a local observable at locations such as the Earth.

Neither angle is directly observable deep in the field due to the lack of any un-deflected reference there. An actual observer at the tangent point would look exactly tangentially to "see" any point on the path of the light ray. Space there is sufficiently curved that it does not match our coordinate system. The displacement angle is a computational tool which at very far distances asymptotically approaches the wave angle, and thus will over the entire path converge to the asymptotic observable angle.

\footnotetext{
1 At current writing, IE or Firefox will paste to spreadsheets correctly, but not Chrome.
} 


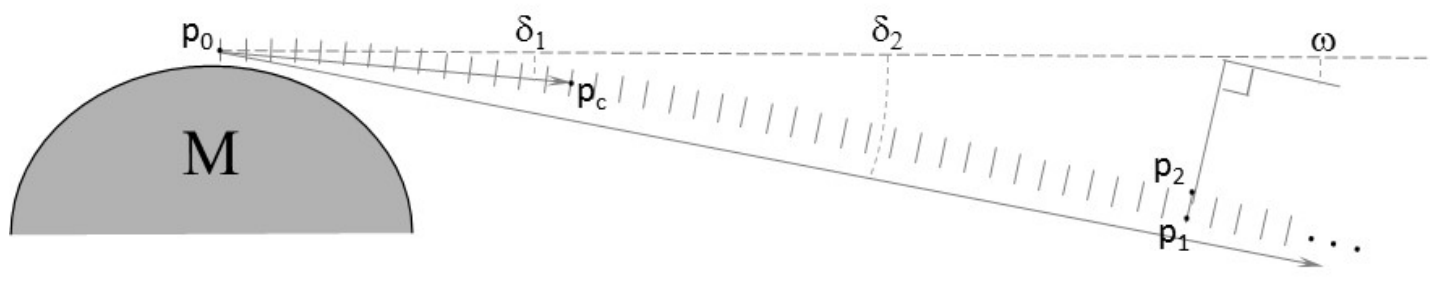

Figure 3. Coordinate displacement and wave angles (1/2 of total angle shown, from limb outward)

\section{$3 \quad$ Results and Discussion}

\subsection{Solar Light Bending \& Method Verification}

The parameters shown in Figure 2, especially initial radius, were chosen for compatibility with the first order analytical light bending value of 1.7504 arc seconds usually given. (Ibid. [8]) In other words, those values will give 1.7504 in the $1^{\text {st }}$ order analytical formula. Table 1 gives the numerical results out to 10 AU (beyond which bending is essentially negligible at the accuracy shown) using time steps as indicated and a simple Euler integrator for the three precisions.

Table 1. Solar light bending computation to $10 \mathrm{AU}$, results in arc seconds (results are total angle, or twice the half-angle of Fig. 3)

\begin{tabular}{llll}
\hline & Javascript (double) & $\mathrm{C}$ (long double) & GCC Quad Precision \\
\hline initial $\Delta t$ & 1 millisecond & 100 nanoseconds & 100 nanoseconds \\
Bending at 10 AU & 1.75043159 & 1.75040894 & 1.75040682 \\
$\mathrm{~N}=1$ Bending & 1.75043149 & 1.75040328 & 1.75040029 \\
\hline
\end{tabular}

Time step sizes were chosen for reasonable accuracy in modest run times. For the double precision web script, time steps significantly less than 1 millisecond result in unreliable results due to round off. This can be determined by varying parameters such as initial radius or solar mass to see if the result varies as expected. However, even with larger step sizes round off can result in oscillation about the final trajectory, making the result of the script version more like $1.7505 \pm 0.0003$. The script is most useful for copy and paste of results to a spreadsheet, but the 128 bit version should be used for accurate results.

Most of the bending occurs near the sun, so for the higher precision cases a schedule of increased step sizes was used to reduce run time. At .5, 5, 20.0, and 100.0 seconds into the simulation the step sizes were adjusted to $.0000002, .000001, .00002$, and .00005 seconds respectively. All results agree well with the published figure of 1.7504 arc seconds $1^{\text {st }}$ order bending. GR $2^{\text {nd }}$ order bending at the limb of the sun is 1.75040681 arc seconds calculated by the method of Bodenner and Will, (Ibid. [8]), showing excellent agreement with the two high precision cases, especially with the 128 bit case. It appears the method works well even at $2^{\text {nd }}$ order, and might be suitable for computations such as light bending by a neutron star. The $\mathrm{C}$ version has parameters for a neutron star, with an iteration loop to plot bending over a range of radii.

There are options to turn off either time dilation or spatial bending, done by adjusting coordinate velocities, and disabling either reduces bending by $1 / 2$. The plot in Figure 4 shows the change in bending angle at logarithmic time increments in light-seconds from closest approach, comparing the full and correct computation (dark bars) with a time dilation only computation (light bars) constructed by using the tangential coordinate velocity in both directions. This is comparable to Einstein's 1911 computation, which is the usual reference point over which bending is considered to be doubled. (Ibid. [6]) It is apparent from the figure that the "factor of 2 " is present at all points on the trajectory when using coordinate deflection angle (b). Using wave front angle (a) the factor of 2 is not present until after .5 to 1 light second, while a much greater factor is present above 6 light seconds. Wave angle bending is 
complete by about 50 light seconds $(0.1 \mathrm{AU})$, and so Earth telescopes register almost the full bending. But the deflection angle bending continues accumulating more than 20 times that far.

Table 2 shows detail of the first two time steps following the tangent position for the quad precision version with the recommended initial time step of 100 nanoseconds. The displacement ratio is $1.9997073684 \pm 0.00000000004$ for these two steps, indicating round off error is quite small and that the bending is different from the exact double by about $.03 \%$, which is due to $2^{\text {nd }}$ order effects (see section 5).
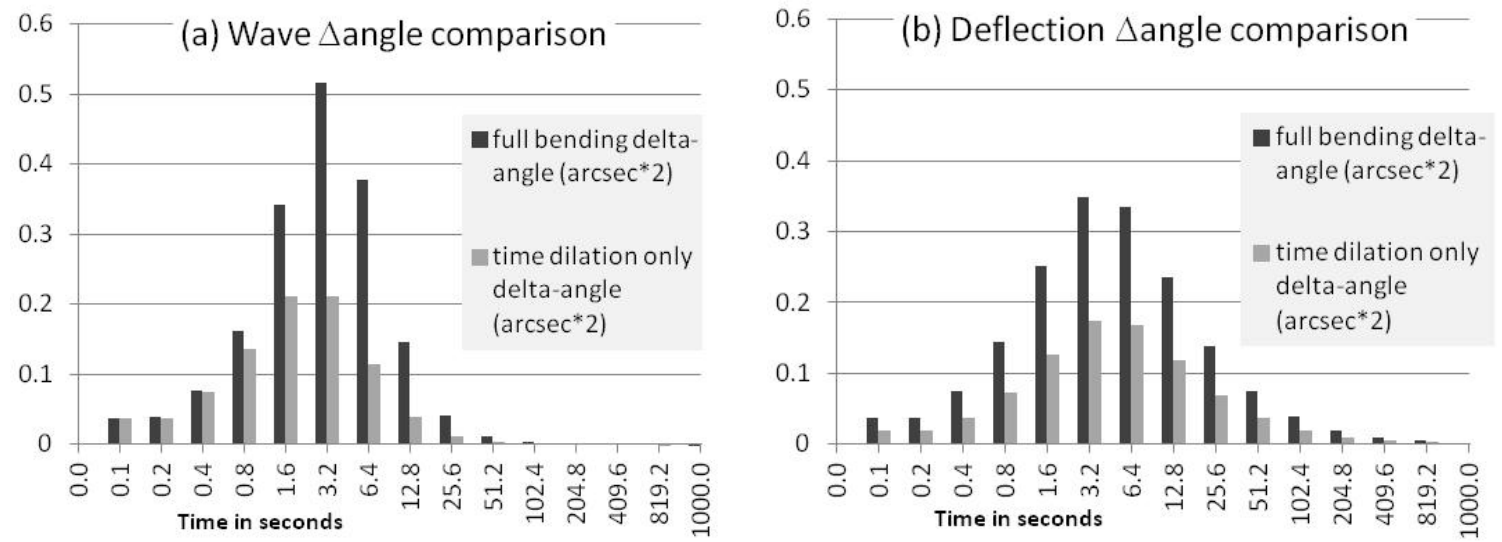

Figure 4. Bending increment ( $\Delta$ angle) vs. light-distance from tangent point

Table 2. Double displacement bending for early iterations for time step of 100 nanoseconds

\begin{tabular}{lllc}
\hline iteration & $\Delta x$ normal bending & $\Delta x$ single bending & ratio \\
\hline 2 & $-5.4768403548 \mathrm{E}-12$ & $-2.7388209097 \mathrm{E}-12$ & $1.9997073687 \mathrm{E}+00$ \\
3 & $-1.6430521062 \mathrm{E}-11$ & $-8.2164627271 \mathrm{E}-12$ & $1.9997073690 \mathrm{E}+00$ \\
\hline
\end{tabular}

Shapiro delay is the excess delay encountered when bouncing radar signals off Venus and timing the round trip when Venus (or Mars) is nearly in opposition due to the combination of time dilation and spatial expansion of the distance. [12] This was calculated by using an average orbital distance and computing the time to the orbital radius for full application of GR coordinate velocities vs. no change in velocities at all (options in the quad precision version), and multiplying the result by 4 since this represents $1 / 4$ of the round trip. Although Shapiro delay is not as sensitive to the closest approach radius as light bending, most of the delay is still near the sun. An excess delay at the solar limb radius of 258.6 microseconds was obtained, comparable to 247 microseconds calculated by Shapiro for an Earth-Mars path, and 180 microseconds for a measured Earth-Venus path at somewhat larger closest approach. The algorithm obtains 195 microseconds for $3.4 \times 10^{6} \mathrm{~km}$ closest approach, and 189 microseconds for $4 \times 10^{6} \mathrm{~km}$ approach.

\subsection{Geometrical Interpretation}

In this section we'll use the approximations $\Upsilon=\left(1-2 G M / r c^{2}\right)^{-1 / 2} \approx\left(1+G M / r c^{2}\right)$ as well as $1 \pm x \approx 1 /(1 \mp x)$ and $\sin x \approx x$ for $x \ll 1$. The important thing demonstrated by the numerical method is that in these coordinates displacement angle bending is everywhere except at the exact tangent point equal to double of the value expected from time dilation alone. It might seem from "intuition" that using the modified Huygens method, nearly horizontal ray bending would be dominated by the tangential coordinate velocity, and as Figure 4(a) and 4(b) show, that is true for wave angle but not for displacement angle. 


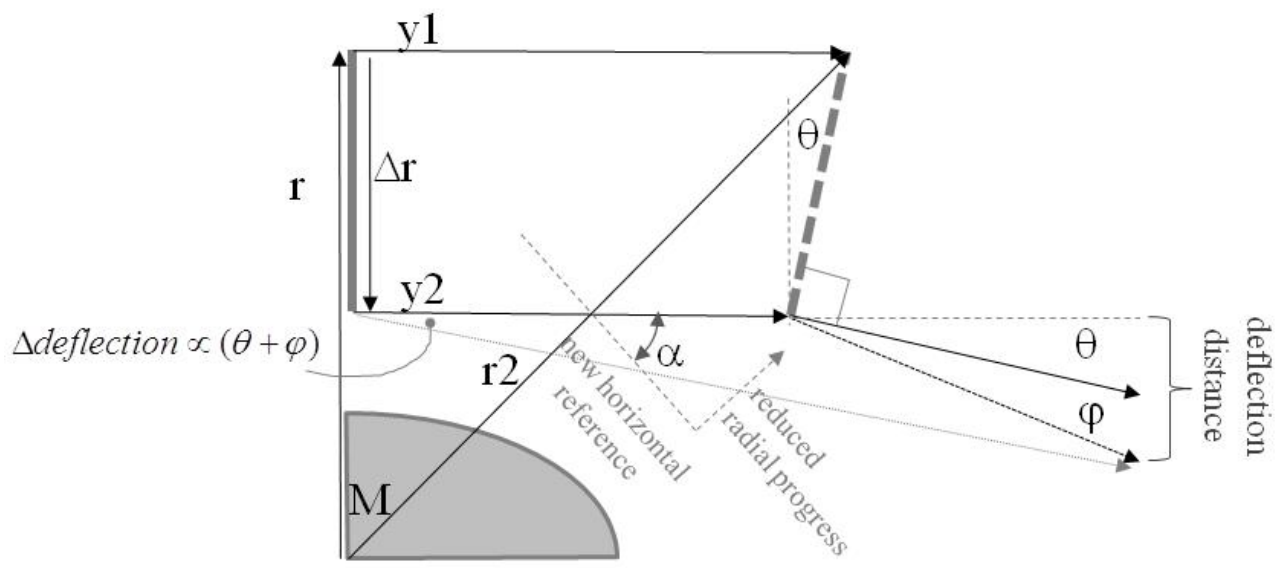

Figure 5. Nearly-tangential ray bending components

Consider Figure 5 showing the propagation of two wave front points at separation $\Delta r$ along displacement vectors $y 1$ and $y 2$. If exactly tangential, the difference in displacement vectors due to the coordinate velocity difference across $\Delta r$ for coordinate time step $\Delta t$ is

$$
\begin{aligned}
& \theta=\sin ([y 1-y 2] / \Delta r) \approx[y 1-y 2] / \Delta r \\
& \Rightarrow \theta \approx[\Upsilon(r) c \Delta t-\Upsilon(r-\Delta r) c \Delta t] / \Delta r \\
& \Rightarrow \theta_{\text {exactly_tangent }} \approx\left(G M / r c^{2}\right) c \Delta t / r
\end{aligned}
$$

If instead we used radial coordinate velocity, as would be approximately true for a ray that had gone far past the tangent point and was traveling almost radially, then the light speed adjustment would be instead the value $\Upsilon^{2} \approx\left(1+G M / r c^{2}\right)^{2} \approx 1+2 G M / r c^{2}$ giving a double bending rate for the nearly radial ray:

$$
\theta_{\text {nearly_radial }} \approx\left(2 G M / r c^{2}\right) c \Delta t / r
$$

These are wave angle bending rates, which we will modify later. What we will do now is show that also a nearly-tangent (instead of exactly tangent) ray is bent double using displacement angles. Since light is an electromagnetic field which can be found by superposition, we can then decompose any ray into nearly tangential and nearly radial basis vectors, and argue that if they both give double bending, then the composite ray is also double bent according to (3). We need only consider half the path, from the tangent point outward, as the other half will be symmetric to that. The horizontal reference in a spherical field for a light ray just passing the closest tangent point is continually falling away, and relative to it the light ray is continually turning up. In Figure 5 the angle $\alpha$ represents the upturned angle of an un-bent ray to the new horizontal. As the light enters the radial direction $r 2$, it encounters the expanded local radial distances, or from a coordinate viewpoint it slows due to the radial coordinate velocity, and makes reduced progress pulling away from the changing horizontal reference, creating the additional bending angle $\varphi$. This new bending $\varphi$ affects all the propagation points on the wave front, and therefore does not affect the wave angle, but does cause a displacement of the ray and affects the deflection angle (shown back to the tangent point at left). At small angles (Figure 5 is greatly exaggerated so that we can read the angles and labels) where $\sin x \approx x$ the net deflection (distance) will be

$$
\text { deflection_distance }=\sin ^{-1}(\theta+\varphi) \approx \theta+\varphi
$$

The angle through which the tangent turns is $\alpha=\sin (y 1 / r) \approx \sin \left(\Upsilon^{-1} c \Delta t / r\right) \approx \Upsilon^{-1} c \Delta t / r$. The newly discovered horizontal reference requires a breakdown of the old tangential coordinate velocity $v=c \Upsilon^{-1}$ into new component velocities $v_{\tan } \approx v \cos \alpha \approx v$ and $v_{r} \approx v \sin (\alpha) \approx v \alpha$. We must reduce $v_{r}$ by $\Upsilon$ to reflect the reduction in radial coordinate velocity over tangential, giving $v_{r}{ }^{\prime} \approx v_{r} \Upsilon^{-1} \approx v \Upsilon^{-1} \alpha$. We can find $\alpha=\sin (y 1 / r) \approx y 1 / r=v \Delta t / r$. To find the deflection angle $\varphi$ we use the difference between $v_{r}$ and $v_{r}^{\prime}$ : 


$$
\begin{aligned}
& \varphi=\sin \left[\left(\Delta t v_{r}-\Delta t v_{r}{ }^{\prime}\right) / \Delta t v_{\tan }\right] \approx\left(v_{r}-v_{r}{ }^{\prime}\right) / v \approx\left(v \alpha-v \Upsilon^{-1} \alpha\right) / v \\
& \Rightarrow \varphi \approx\left[1-\Upsilon^{-1}\right] \alpha \approx\left[1-\Upsilon^{-1}\right] v \Delta t / r \approx\left[1-\Upsilon^{-1}\right] c \Upsilon^{-1} \Delta t / r \\
& \Rightarrow \varphi \approx\left[1-\left(1-G M / r c^{2}\right)\right] c \Delta t / r \approx\left(G M / r c^{2}\right)\left(1-G M / r c^{2}\right) c \Delta t / r \\
& \Rightarrow \varphi \approx\left[\left(G M / r c^{2}\right)-\left(G M / r c^{2}\right)^{2}\right] c \Delta t / r \approx\left(G M / r c^{2}\right) c \Delta t / r
\end{aligned}
$$

Since (3) is also the time dilation only bending, and matches (5), we have analytical verification that $\theta \approx \varphi$, and the near-tangential deflection bending angle, proportional to $\theta+\varphi$, is the double of the amount due only to time dilation. To reach this conclusion we ignored a $2^{\text {nd }}$ order term, so the "double bending" is only a first order approximation and not quite true when $2^{\text {nd }}$ order is considered.

In the nearly tangential case we have exactly what we need - a conclusion that deflection bending is exactly doubled. In the exactly radial direction there is of course no bending at all, so in that direction also we specify a basis vector which is "nearly radial." In that case one of the wave front points is slightly below the other by $\Delta r$, moving slightly slower as a result, and thus the wave angle is rotating. While this $\Delta r$ is in a different direction with respect to the wave front, nevertheless the same algebra used in (2) and (3) applies, and so we have the result that due to the radial coordinate velocity difference the wave angle is changing at the double of the rate it would be from time dilation alone (tangential coordinate velocity difference).

Small wave angle changes will result in displacement changes in the same proportion eventually as the ray continues, i.e. also doubled, meaning we can take the wave angle as a proxy for eventual displacement angle bending. So we conclude we can make near-radial and near-tangential basis vectors for an arbitrary light ray, and treat the component velocities exactly as we have treated the nearly vertical and horizontal ray velocities, with the result that the composite light ray is always accumulating deflection bending at the double of the rate attributed to time dilation only. We cannot make definite statements about wave angle and therefore measured bending at finite radii from this argument, only about total asymptotic bending.

\subsection{Double Bending, Curvature and Equivalence}

A better and equally simple method to understand near-radial bending is to simply realize that due to the expanded radial distance, if equivalence frames (a rigid elevator for example) are assumed to be of fixed proper dimensions, there will be more of them in the radial direction by the spatial expansion factor. Therefore there is no extra bending in any one of them. Instead the extra bending is due to an expanded number of them in a global distance, confirming Ehlers and Rindler's differentiation between local and global bending. If we compute bending in local proper units, y1 and y2 will be larger by the spatial factor $\Upsilon$, so we again have $\Upsilon^{2}$ as the adjustment factor and the result (3) applies.

Therefore double bending does not in any way imply double falling or a violation of the EP. From Figure 4(a) we conclude a light ray at the limb of the sun has traveled 100,000 miles and is progressing upward at an angle of around 8 degrees before noticeable deviation from single bending wave angles occurs. Before that, it is essentially indistinguishable from a near-horizontal ray in a rocket or elevator. The displacement angle viewpoint reveals that the doubling effect is associated with travel in the radial direction associated with spatial curvature.

\subsection{Testing Metrics with Light Paths Near a Neutron Star}

To illustrate investigation of an extra-solar question of fundamentals, we examine light bending near a neutron star of 1.4 solar masses with a closest approach radius from 12 kilometers (assumed to be approximately the size of the star) to 32 kilometers, and compare GR-Schwarzschild bending to an alternate metric, arbitrarily selected to match GR except at $2^{\text {nd }}$ order and to have some interesting property, such as the event horizon at a different location. In this case, we use $\Upsilon=\left(1+G M / r c^{2}\right)$ exactly, not an approximation. We are not concerned here with the justification or selection of any particular alternate metric, but with an investigation of whether neutron star light bending could be used to distinguish these two very close metrics. We name them by the multiplier of the GM term, namely $\mathrm{n}=-2$ for $\mathrm{GR}$, and $\mathrm{n}=1$ for our comparison. Figure 6 shows the light bending from the first order analytic formula and from our algorithm for each of the two. 


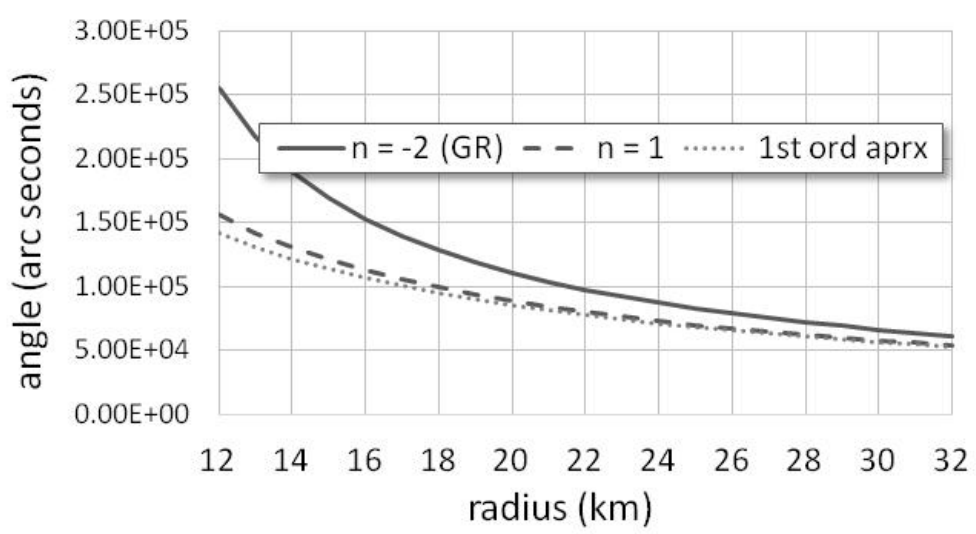

Figure 6. Light bending near a 1.4 solar mass neutron star for two metrics

A common problem in second order measurements is that one does not know the masses or even physical parameters such as $\mathrm{G}$ well enough to reach a conclusion about a single measurement point. It is apparent from the chart, however, that if a range of data were obtained, then the shape of the curve for the two metrics would be easily distinguishable. This is not true in the solar system where the difference between the two metrics is only 6 parts per million. At present we have some expectation of being able to do microarcsecond interferometry, [13] which only has kilometer resolution out to about a light year, but as time goes on the experiment may become possible.

\section{Conclusions}

By using nearly vertical and nearly horizontal light rays, we are able to produce a diagrammatic explanation of light bending with an analytic confirmation that uses only simple algebra and a very small amount of trigonometry. These insights were gained by studying a numerical simulation in a Euclidean coordinate space, with reference to the underlying space-time geometry via a Schwarzschild line element used to calculate coordinate velocity. A least-time two-point wave propagation methodology effectively finds the geodesic directly from the metric. It is easy to ask "what if" questions either about theoretical matters or experimental observables. As an analysis tool not tied to a particular formulation of gravity it can be used to quickly compare effects and evaluate possibilities for experimental tests.

Theoretical insights obtained include first that using deflection angle in the distant observer coordinates, which is congruent with the asymptotic observable angle, the coordinate deflection is double at every time step, with an analytical demonstration confirming this. One could in principle plot the un-deflected path in real space using Schwarzschild coordinates, as circumferences are measurable by all observers. (The geodesic would appear straight, and the hypothetical single-bent path would not.) But it appears that gravity modifies the light path over theoretical un-bent and single-bent paths by double deflection at each point, while wave angles (or trajectories) follow the traditionally early vs. late pattern of Figure 4(a). Additionally it was determined that the spatial bending occurs due to expanded radial distance.

An experimental insight obtained was that a factor of 100 to 1000 in angular resolution is required before $2^{\text {nd }}$ order field strength tests using objects such as neutron stars are possible, based on the shape of the bending vs. radius relation. There has been a $1 \times 10^{6}$ improvement already in the last half century, and such a capability would have many other uses as well.

\section{References}

1. Ying, L., Candes, E.J., "Fast Geodesics Computation with the Phase Flow Method," Jour. of Comp. Phys., 220, 1 , pp 6-8 (2006). 
2. Vincent, F.H., Gourgoulhon, E. and Novak, J., "3+1 geodesic equation and images in numerical spacetimes," Class. Quantum Grav., 29, 245005, 1-17 (2012).

3. Karas, V., Vokrouhlicky, D., Poinarev, A.G., "In the vicinity of a rotating black hole: a fast numerical code for computing observational effects," MNRAS, 259, 3, pp 569-575 (1992).

4. Einstein, A., Relativity: The Special and General Theory, Methuen 86 Co. Ltd., London, UK (1916), trans. Lawson, R., Henry Holt \&S Co., NY (1920), p. 153.

5. Ehlers, J. and Rindler, W., "Local and Global Light Bending in Einstein's and other Gravitational Theories," General Relativity and Gravitation, 29, 4 (1997).

6. Einstein, A., "On the Influence of Gravitation on the Propagation of Light," Annalen der Physik, 35 (1911).

7. Einstein, A., "The Foundation of the General Theory of Relativity," Annalen der Physik, 49 (1916).

8. Bodenner, J. and Will, C., "Deflection of light to second order: A tool for illustrating principles of general relativity," Am. J. Phys., 71, 8 (2003).

9. Taylor, J.H., Fowler, L.A. and McCulloch, P.M., "Measurements of general relativistic effects in the binary pulsar PSR1913+16," Nature, 277, 8, pp. 437-440 (1979).

10. Misner, C.W., Thorne, K.S. and Wheeler, J.A., Gravitation, W.H. Freeman 83 Co., San Francisco, p. 3 (1973).

11. Kassner, K., "Classroom reconstruction of the Schwarzschild metric," Eur. J. Phys., 36, 6, pp. 31-50 (2015).

12. Shapiro, I.I., Pettengill, G.H., Ash, M.E., Stone, M.L., Smith, W.B., Ingalls, R.P. and Brockelman, R.A., "Fourth Test of General Relativity: Preliminary Results," Phys. Rev. Lett., 20, 1265 (1968).

13. Kopeikin, S.M. and Makarov, V.V., "Gravitational bending of light by planetary multipoles and its measurement with microarcsecond astronomical interferometers," Phys. Rev. D, 75, 6, p. 062002 (2007) 